\title{
Entornos de movilidad peatonal: una revisión de enfoques, factores y condicionantes
}

Luis Miguel Valenzuela-Montes. Universidad de Granada, Granada, España. Rubén Talavera-García. Universidad de Granada, Granada, España.

RESUMEN | El peatón ocupa un lugar destacado en la movilidad urbana, tanto por representar el modo de transporte más básico y que alimenta al resto de modos de transporte, como por mantener una relación intensa y directa con las actividades urbanas, conformando los denominados entornos de movilidad peatonal o entornos peatonales. Es por ello que profundizar en el conocimiento de aquellos factores de los entornos construidos que mayor relación guardan con el peatón resulta fundamental, tanto para mejorar la calidad de la movilidad peatonal en las calles, como para lograr que las centralidades, el transporte público o los espacios públicos sean más accesibles. Con esta finalidad, el presente artículo evalúa, a través de la bibliografía especializada, los enfoques más frecuentes de análisis de la figura del peatón, así como los factores que son tenidos en cuenta por la población para optar por la modalidad de transporte que él representa, considerando su dimensión y su valoración.

PALABras ClaVe | espacio público, medioambiente urbano, movilidad.

ABSTRACT | Pedestrians play an important role in the urban mobility system, as walking is the most fundamental mode of transportation, and is used to connect with other modes of transportation. Furthermore, pedestrian mobility has a strong relationship with the built environment, thus conforming what is known as pedestrian mobility environments or pedestrian environments. In this way, it is necessary to achieve a better understanding of the factors that encourage people to walk as a form of viable transportation in the city, and improve the quality of mobility when pedestrians attempt to access central urban areas, public places or public transport hubs. With this objective in mind, the paper evaluates the most common analyses regarding pedestrian mobility, through a review of specialized references in the literature, including the factors considered in these references, and the various dimensions and valuations involved in the decisions by the population to opt for walking as a preferred mode of transportation.

KEY WORDS | public space, urban environment, mobility.

Recibido el 28 de agosto de 2013, aprobado el 28 de julio de 2014

E-mail: Luis Miguel Valenzuela-Montes, lvmontes@ugr.es | Rubén Talavera-García, rtalaverag@ugr.es

Correspondencia: Escuela Técnica Superior de Ingeniería de Caminos, Canales y Puertos, Campus de Fuentenueva, s/n, 18071 Granada, España 


\section{Introducción}

La figura del peatón posee una fuerte relevancia en las ciudades, dada su doble faceta: la de habitante y la de usuario del modo de transporte más básico. Es por esta doble faceta que se puede considerar la "peatonalidad" como el modo de transporte que mantiene una relación directa e intensa entre el habitante urbano y la ciudad a través de los sentidos, a la vez que le permite interaccionar con otros peatones (Gehl, 1971; Peters, 1981), participar de la actividad comercial y cultural en las calles (Venturi, Brown \& Izenour, 1977), o apreciar el entorno natural y arquitectónico (Jacobs, 1993). En definitiva, el peatón, dada su relación con el medio urbano, puede apreciar las características singulares de las rutas por las que se desplaza, haciendo que cada una tenga identidad propia (Lynch, 1960).

El entorno urbano desempeńa un papel fundamental en la movilidad peatonal, ya que la presencia o no de determinados elementos a lo largo de las calles, así como las características físicas que les son propias, pueden potenciar los desplazamientos peatonales, o disuadirlos. En el marco de esta concepción, se conoce con el término de "entorno peatonal" aquel en el cual existe una predominancia de desplazamientos a pie (Borst, Vries, Graham, Van Dongen, Bakker \& Miedma, 2009; Zacharias, 2001), basada en la presencia de factores que promueven la movilidad peatonal.

Desde este planteamiento tiene significado llevar a cabo una revisión extensa de la bibliografía especializada existente para conocer el estado del arte de la movilidad y accesibilidad peatonal, para así poder comprender con mayor profundidad cuáles son los principales enfoques que existen, los principales factores considerados dentro de cada enfoque y la dimensión a la que pertenecen dichos factores. Del mismo modo, es interesante comprender la jerarquía que posee cada uno de los factores considerados dentro del conjunto de factores que influyen en la movilidad peatonal, teniendo en cuenta el contexto en el cual son llevados a cabo cada uno de los estudios analizados.

Así pues, podría considerarse a priori que la identificación de factores relacionados con los viajes peatonales viene a ser una necesidad para llevar a cabo una integración efectiva de la estructura urbana y de los patrones de viaje en el marco de la planificación y gestión de la movilidad (Correa-Díaz, 2010), en especial de la movilidad no motorizada (Clifton, Livi Smith \& Rodriguez, 2007). Además, en última instancia, una mejora en los métodos para la planificación de la movilidad peatonal repercute en facilitar el acceso al transporte público (Rodríguez, Brisson \& Estupiñán, 2009), así como a diferentes centralidades y espacios públicos. Incluso cabe considerar que solo en la medida en que una ciudad garantice que sus habitantes se benefician del acceso a tales destinos, potenciando además la interacción social (Hernández, 2012), podrá clasificársela de exitosa (Dávila, 2012) en materia de movilidad y desarrollo urbano.

En esta línea, el presente artículo pretende ser la base sobre la cual construir un modelo de entornos de movilidad peatonal, dada la necesidad de progresar en el diseño de indicadores y procedimientos más adecuados para identificar entornos urbanos peatonales a través de la interpretación y comprensión de su funcionamiento; y de aportar así mayor rigor en el proceso de planificación respecto a la adopción de medidas o estrategias concretas. 
Con tal finalidad, se propone una revisión del estado del arte en los temas vinculados a la movilidad peatonal, en la cual se lleva a cabo una evaluación del contenido de la literatura seleccionada.

\section{Metodología}

La necesidad de profundizar en el conocimiento de los entornos urbanos y su relación con la movilidad peatonal da lugar a que se proponga el proceso metodológico que se ilustra en la Figura 1, que parte de un proceso de revisión y selección de referencias bibliográficas. El conjunto de referencias seleccionado constituye la base sobre la cual llevar a cabo una evaluación del contenido de cada una de las referencias en tres fases. En la primera, más general, se evalúa el enfoque predominante a la hora de considerar los desplazamientos del peatón. En una segunda fase se profundiza en cada una de las referencias, identificando los factores evaluados en función de sus respectivos enfoques. Finalmente, se propone una tercera fase, en la cual se indaga -dentro de las referencias seleccionadas- el peso que posee cada uno de los factores evaluados respecto al resto, valor determinado ya sea a través de la opinión de ciudadanos o de expertos.

\section{Selección de referencias}

Dado que el núcleo fundamental sobre el que se estructura el presente artículo es la bibliografía científica existente en relación con la temática tratada, se ha realizado un proceso de búsqueda selectiva de referencias a partir de tres criterios: proximidad temática, palabras clave e impacto de la referencia.

En primer lugar, se ha tenido en cuenta la proximidad temática entre la investigación desarrollada en las diferentes obras revisadas y el contexto del presente artículo. En este sentido, se pueden distinguir diferentes perspectivas desde las cuales se enfoca la figura del peatón. Una de las más tradicionales es la que toma como punto de referencia el transporte o la geografía, evalúa la accesibilidad a diferentes lugares en función de la distancia o tiempo necesario para alcanzarlos y, sobre esa base, promueve equidad en la accesibilidad (Neutens, Schwanen, Witlox \& De Maeyer, 2010; Talen \& Anselin, 1998). Desde la arquitectura, se ha prestado especial atención a los espacios públicos -incluyendo las calles- como lugares dinamizadores de la ciudad (Gehl, 1971), lugares de socialización, de comercio, etcétera, en los que se otorga especial importancia a los diferentes elementos que favorecen dichos procesos. No obstante, vinculada a esta perspectiva del diseño urbano, se han desarrollado nuevas líneas de investigación más afines a campos de la medicina y la psicología que a los estudios urbanos, en los que se focaliza sobre la actitud de la población frente al caminar y en la forma en que los elementos presentes en los diferentes espacios públicos influyen en dicha actitud. Esta línea de investigación novel está muy relacionada con la creciente preocupación en el campo de la salud pública por los estilos de vida sedentarios (Lee, Mama, Medina, Ho \& Adamus, 2012; World Health Organization, 2011). 
FIGURA I | Proceso de revisión, selección y clasificación de referencias

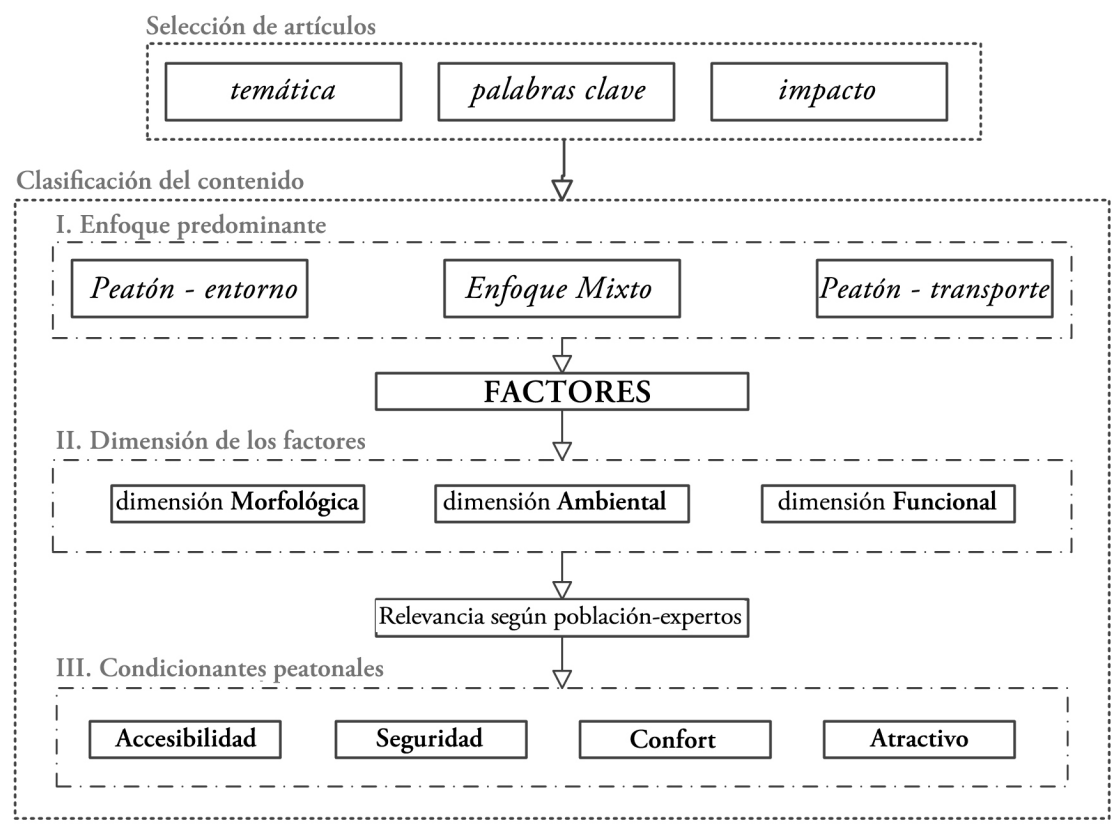

FUENTE ELABORACIÓN PROPIA

Estas perspectivas en el estudio de la figura del peatón van a acotar las áreas de conocimiento en las que realizar la búsqueda de referencias, de manera que se exploran las principales bases de datos -Journal Citation Report (JCR) y Scimago Journal and Country Rank (SJR)-, teniendo en cuenta aquellas áreas de conocimiento que son más afines a la evaluación del presente estudio (Cuadro 1). Se establece, además, un arco temporal de 1995 a la actualidad. Este intervalo se corresponde aproximadamente con una evaluación de las últimas dos décadas, lo que posibilita detectar la evolución de los enfoques y perspectivas y las innovaciones en ellos, permitiendo además establecer cuatro subintervalos de cinco años con los que hacer comparativas temporales. Una vez seleccionadas las bases de datos en las que se va a realizar la búsqueda y fijada la temática, el intervalo temporal de búsqueda y las áreas de conocimiento más afines, se lleva a cabo una búsqueda teniendo en cuenta los dos criterios restantes: palabras clave e impacto. Las palabras clave relacionadas con la movilidad peatonal son dos términos anglosajones principales: walk y pedestrian. No obstante, además son consideradas las palabras derivadas de ambas, como quedan recogidas en el Cuadro 1. 
CUADRo I | Variables tenidas en cuenta para la selección de referencias

\begin{tabular}{|l|l|l|}
\hline \multirow{4}{*}{ PALABRAS CLAVE } & Walk y derivados (walkability, walking, walking environment) \\
\cline { 2 - 3 } & Caminar: caminabilidad, caminar, entornos caminables \\
\cline { 2 - 3 } & Pedestrian y derivados (pedestrian environment) \\
\cline { 2 - 3 } & Peatón: entornos peatonales \\
\cline { 2 - 3 } & Access y derivados (accessibility) \\
\cline { 2 - 3 } BASES DE DATOS & Acceso: accesibilidad & Scimago Journal and Coutry Rank (sJR) \\
\hline \multirow{2}{*}{$\begin{array}{l}\text { ÁREA DE } \\
\text { CONOCIMIENTO }\end{array}$} & Social Sciences Edition & Social Sciences \\
\cline { 2 - 3 } & \multirow{2}{*}{ Science Edition } & Environmental Science \\
\cline { 2 - 3 } & & Psychology \\
\cline { 2 - 2 } & & Engineering \\
\cline { 2 - 2 } & & Medicine \\
\hline
\end{tabular}

FUENTE ELABORACIÓN PROPIA

Establecidos los parámetros para la búsqueda y selección de referencias, es preciso analizar el contenido de las mismas. Para ello se propone un proceso basado en tres fases, que va profundizando en el contenido de las diferentes referencias.

Fase I. La primera fase de la metodología presentada pretende identificar el enfoque con el que es considerada y analizada la figura del peatón en cada una de las referencias, ya sea considerando el caminar como un modo de transporte y, por tanto, capaz de conectar un origen con un destino, reduciéndose u obviándose las características del trayecto; o un enfoque en el que se da una mayor relevancia al entorno por el cual transita el peatón y, por tanto, poniendo énfasis en caracterizar con mayor detalle el trayecto, sin considerar si tiene un origen o un destino. Se considera también un enfoque mixto en el que, en mayor o menor medida, ambas perspectivas sobre los trayectos peatonales son tenidas en cuenta.

Fase II. Evaluadas las referencias según su enfoque, en una segunda fase se profundiza en cada una de las referencias, con el fin de recoger los diferentes factores urbanos considerados. De esta manera se puede apreciar qué factores se examinan con más frecuencia en cada uno de los enfoques evaluados en la fase anterior. Posteriormente, todos los factores son evaluados según la dimensión a la que contribuyen. En este sentido, las dimensiones que dan lugar al "sentido de lugar" propuestas por Montgomery (1998) se han adaptado a la relación entre diseño urbano y movilidad peatonal, renombrando las tres dimensiones como morfológica, funcional y k. Esta evaluación permite distinguir cómo los diferentes enfoques relacionados con la movilidad peatonal prestan mayor o menor atención a las distintas dimensiones que dan lugar a los diferentes "sentidos de lugar".

Fase III. Finalmente, la última fase de la metodología incide sobre la importancia relativa de cada uno de los factores recogidos en el desplazamiento peatonal. Para ello, del total de referencias seleccionadas se descartan aquellas que no llegan a este nivel de detalle. Con esta segunda selección de referencias se evalúan los factores según su relevancia respecto del conjunto. Dicha evaluación tiene lugar sobre la 
base de los resultados obtenidos en las encuestas a población general o específica y a paneles de expertos. No obstante, para poder analizar de manera conjunta y comparada la valoración de factores, es necesario homogeneizarlos. Este proceso de homogeneización se realiza pasando de diferentes factores estadísticos a una escala simplificada de 0 a 10 , en la que el grado de influencia del factor varía entre la mínima influencia (0) y la máxima influencia (10).

Una vez completadas las tres fases y con una visión en profundidad de los enfoques y factores relacionados con la movilidad peatonal en entornos urbanos, se discutirá sobre los resultados obtenidos de aplicar la presente metodología.

\section{Caracterización de los entornos urbanos respecto a la movilidad peatonal}

Seleccionadas las referencias siguiendo el proceso descrito en el apartado relativo a la metodología (Cuadro 2), se procede con el análisis de las mismas según las tres fases propuestas, yendo progresivamente de una evaluación más superficial a una de mayor detalle.

Fase I: Enfoques de la movilidad peatonal en entornos urbanos

La primera de las fases relativa a la evaluación de las referencias seleccionadas según el enfoque que tengan en relación con la movilidad peatonal en el entorno urbano se muestra a continuación (Cuadro 2). En dicho cuadro se puede apreciar cómo, en el conjunto de referencias, los enfoques se dan en porcentajes similares respecto del total. Así, el enfoque que ocupa el primer lugar según su porcentaje respecto al total es el enfoque mixto, con un 36\%; lo sigue el enfoque peatón-entorno, con un 34\%; y finalmente el enfoque peatón-transporte, con un $26 \%$.

CUADRO 2 | Referencias bibliográficas seleccionadas ordenadas alfabéticamente

\begin{tabular}{|c|c|c|c|}
\hline \multirow[b]{2}{*}{ REFERENCIAS } & \multicolumn{3}{|c|}{ ENFOQUE } \\
\hline & $\begin{array}{c}\text { PEATÓN } \\
\text { TRANSPORTE }\end{array}$ & $\begin{array}{c}\text { PEATÓN } \\
\text { ENTORNO }\end{array}$ & мIXто \\
\hline (Adkins, Dill, Luhr, \& Neal, 2012) & & • & \\
\hline (M. A. Alfonzo, 2005) & & & - \\
\hline (M. Alfonzo, Boarnet, Day, McMillan \& Anderson, 2008) & & & - \\
\hline (Apparicio \& Seguin, 2006) & • & & \\
\hline (Bentley, Jolley \& Kavanagh, 2010) & & & • \\
\hline (Boarnet, Joh, Siembab, Fulton \& Mai Thi Nguyen, 2011) & - & & \\
\hline (Borst et al., 2009) & & & • \\
\hline (Bricka, Sener, Dusza, Wood \& Hudson, 2012) & & & • \\
\hline (Cao, Handy \& Mokhtarian, 2006) & & - & \\
\hline (Cervero \& Kockelman, 1997) & & & • \\
\hline (Clifton et al., 2007) & & - & \\
\hline (Dixon, 1996) & & - & \\
\hline (Ewing \& Handy, 2009) & & • & \\
\hline
\end{tabular}


(continuación)

\begin{tabular}{|c|c|c|c|}
\hline \multirow[b]{2}{*}{ REFERENCIAS } & \multicolumn{3}{|c|}{ ENFOQUE } \\
\hline & $\begin{array}{c}\text { PEATÓN } \\
\text { TRANSPORTE }\end{array}$ & $\begin{array}{c}\text { PEATÓN } \\
\text { ENTORNO }\end{array}$ & MIXTO \\
\hline (Foltête \& Piombini, 2007) & & • & \\
\hline (Foster, Giles-Corti \& Knuiman, 2011) & & - & \\
\hline (Gallimore, Brown \& Werner, 2011) & & & - \\
\hline (Giles-Corti \& Donovan, 2002) & & & $\bullet$ \\
\hline (Giles-Corti et al., 2005) & $\bullet$ & & \\
\hline (Guo, 2009) & & & $\bullet$ \\
\hline (Iacono, Krizek \& El-Geneidy, 2010) & $\bullet$ & & \\
\hline (Jiang, Christopher Zegras \& Mehndiratta, 2012) & & & $\bullet$ \\
\hline (Kelly, Tight, Hodgson \& Page, 2011) & & $\bullet$ & \\
\hline (Koh \& Wong, 2013) & & & $\bullet$ \\
\hline (Landis, Vattikuti, Ottenberg, McLeod \& Guttenplan, 2001) & & $\bullet$ & \\
\hline (Learnihan, Van Niel, Giles-Corti \& Knuiman, 2011) & & & • \\
\hline (C. Lee \& Moudon, 2006) & $\bullet$ & & \\
\hline (S. Lee, Lee, Son \& Joo, 2012) & & & $\bullet$ \\
\hline (Leslie et al., 2005) & & $\bullet$ & \\
\hline (Litman, 2003) & $\bullet$ & & \\
\hline (Lotfi \& Koohsari, 2009) & $\bullet$ & & \\
\hline (Loutzenheiser, 1997) & $\bullet$ & & \\
\hline (Manaugh \& El-Geneidy, 2011) & $\bullet$ & & \\
\hline (McCormack, Cerin, Leslie, Du Toit \& Owen, 2008) & $\bullet$ & & \\
\hline (Millington et al., 2009) & & • & \\
\hline (Moniruzzaman \& Paez, 2012) & & • & \\
\hline (Olszewski \& Wibowo, 2005) & & & $\bullet$ \\
\hline (O'Sullivan \& Morrall, 1996) & $\bullet$ & & \\
\hline (Owen, Humpel, Leslie, Bauman \& Sallis, 2004) & & • & \\
\hline (Parks \& Schofer, 2006) & & $\bullet$ & \\
\hline (Pasaogullari \& Doratli, 2004) & $\bullet$ & & \\
\hline (Pikora, Giles-Corti, Bull, Jamrozik \& Donovan, 2003) & & $\bullet$ & \\
\hline (Pooley et al., 2011) & & $\bullet$ & \\
\hline (Rodríguez et al., 2009) & & & $\bullet$ \\
\hline (Shay, Rodriguez, Cho, Clifton \& Evenson, 2009) & & • & \\
\hline (Shriver, 1997) & & & $\bullet$ \\
\hline (Talen, 2002) & $\bullet$ & & \\
\hline (Townsend \& Zacharias, 2010) & $\bullet$ & & \\
\hline (Weinstein, Schlossberg \& Irvin, 2008) & & & $\bullet$ \\
\hline (Werner, Brown \& Gallimore, 2010) & & & $\bullet$ \\
\hline (Wood, Frank \& Giles-Corti, 2010) & & • & \\
\hline (Zook, Lu, Glanz \& Zimring, 2012) & & & • \\
\hline
\end{tabular}

FUENTE ELABORACIÓN PROPIA 
Ahora bien, además de construir una visión general del enfoque propio de las referencias, resulta conveniente analizar la evolución de cada uno de los enfoques a lo largo de los últimos veinte años. Así, pues, el Gráfico 1 muestra cómo el número de referencias que examinan la figura del peatón respecto a su movilidad ha aumentado constantemente. Además, siguiendo la evolución de cada enfoque, se aprecia cierta homogeneidad en el intervalo 1996-2000 respecto al número de referencias medio anual entre los tres enfoques, aunque con niveles muy bajos. En el intervalo 20012005 se muestra cómo los enfoques comienzan a diferenciarse en cuanto al número de referencias en que aparecen, con un incremento más acentuado de aquellos más específicos, esto es, enfoque peatón-entorno y enfoque peatón-transporte. Sin embargo, esta tendencia varía en el periodo 2006-2010, donde dichos enfoques más específicos se estabilizan en su crecimiento, mientras que el enfoque mixto comienza a ganar relevancia con un número de referencias media anual superior al de los dos enfoques restantes. Dicha tendencia continúa de manera más marcada en el periodo actual, 2011-2015, en el que siguen aumentando las referencias con enfoque mixto, mientras que el enfoque del peatón-entorno tiene un aumento menor y el enfoque peatón-transporte decrece en número de referencias respecto al periodo anterior.

\section{GRÁfICO I Evolución temporal de los distintos enfoques según el número de referencias medio anual}

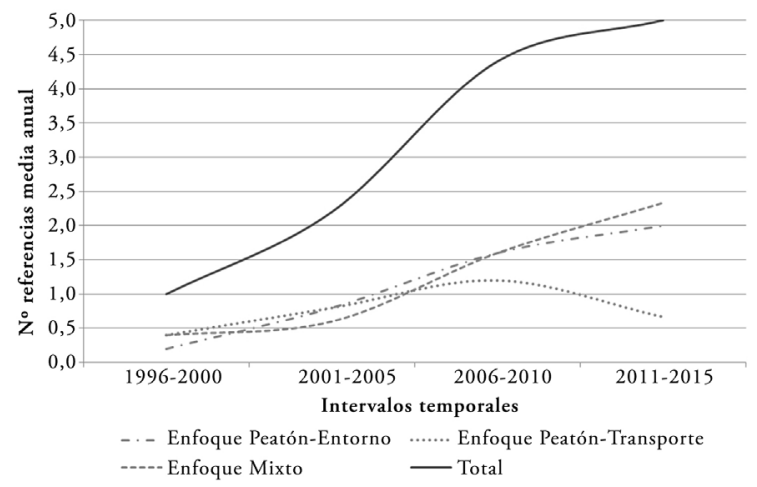

FUENTE ELABORACIÓN PROPIA

La evolución de los tres enfoques muestra, por tanto, que se requiere cada vez más una visión transversal de ellos, como respuesta creativa a la necesidad de abarcar el espacio que queda entre las metodologías presentes más deterministas a través de la creación de metodologías mixtas. Asimismo, resulta relevante el notable incremento anual de referencias sobre esta temática (en cualquiera de los tres enfoques), lo que indica el peso progresivo del peatón en el análisis de la accesibilidad urbana y, por ende, en las propuestas de diseño y gestión de la movilidad urbana.

Fase II: Factores de los entornos urbanos relacionados con la movilidad peatonal Profundizando en las referencias seleccionadas, se ha recogido un total de 48 factores presentes en ellas. Algunos han sido homogeneizados en cuanto a la nomenclatura 
o la denominación, aunque respetándose en todo momento su sentido original. Esta identificación de factores y, sobre todo, la evaluación de su presencia resultan útiles para interpretar y diseñar entornos peatonales según los contextos y objetivos preponderantes de la relación entre movilidad urbana y espacio público.

En lo que respecta a los factores recogidos (Gráfico 2), se puede observar la presencia de algunos relativos a un mismo elemento, como es el caso de la acera o del tráfico, en los cuales se identifican aspectos diferenciados. Del mismo modo, también es constatable la existencia de factores más generales, como sucede con el factor "usos del suelo", que podría contener diferentes aspectos, como "horario", "tipología”, etcétera.

\section{GRÁfICO 2 $\mid$ Presencia de los factores respecto al total de referencias}

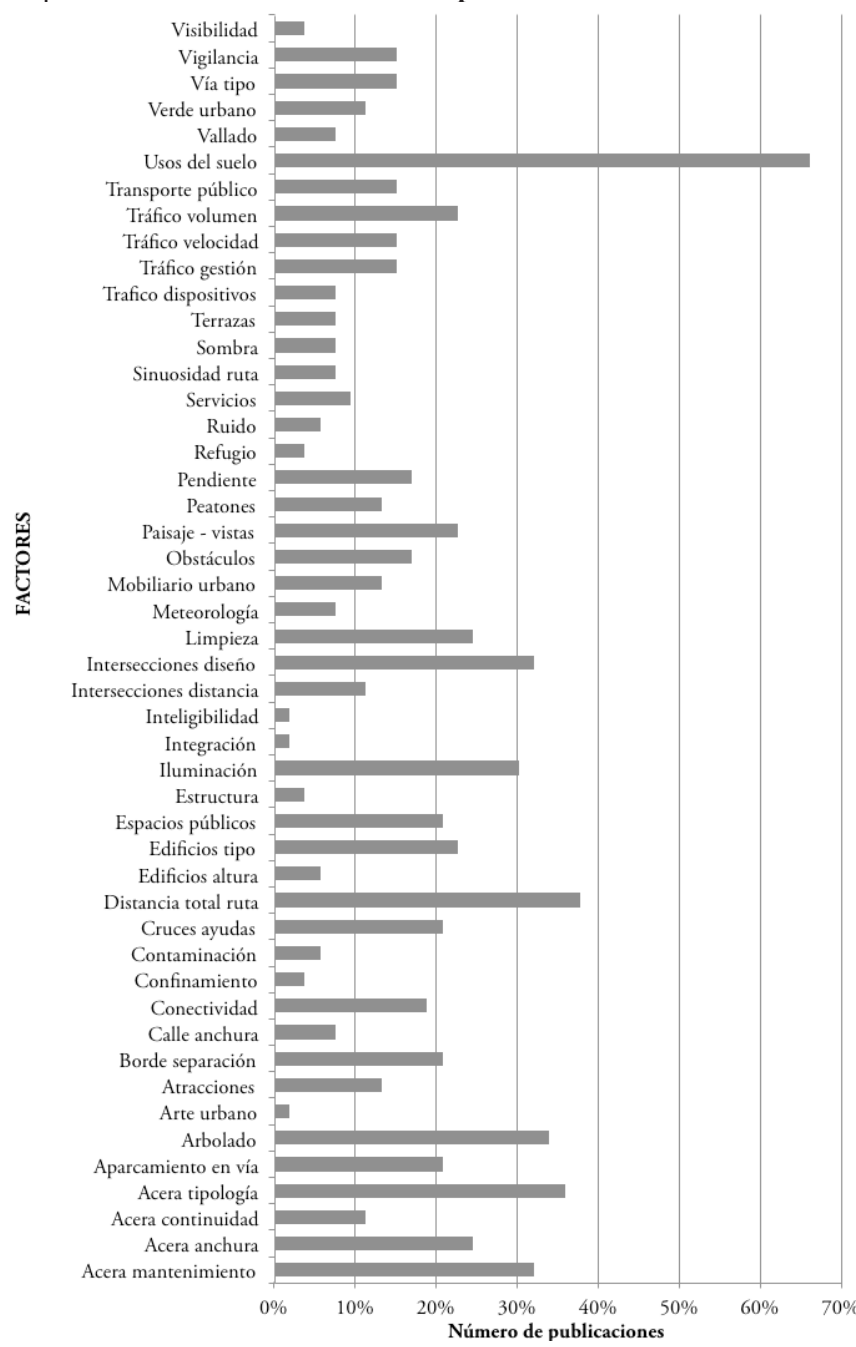

FUENTE ELABORACIÓN PROPIA 
Además de la apreciación anterior, se puede observar la presencia de factores que se encuentran más ligados a análisis cualitativos, como, por ejemplo, la "limpieza" o la "vigilancia", y otros que tradicionalmente son analizados de forma cuantitativa, como la "anchura de la calle", el "volumen de tráfico", etcétera.

Respecto a la frecuencia de aparición de los factores, las diferencias entre unos y otros son constatables. En el extremo superior, con mayor frecuencia se encuentran factores como "usos del suelo", que está presente en más del 65\% de las referencias; o la "distancia total de la ruta" y "acera tipología", con porcentajes superiores al $35 \%$. Estos pueden ser considerados como factores básicos para el estudio de los desplazamientos peatonales. Por el contrario, aquellos con menor presencia son factores con una mayor especificidad, como la "visibilidad", "inteligibilidad", "integración”, "estructura” o "arte urbano”, con porcentajes próximos al 0\%, lo que da a entender que son tratados únicamente por autores con líneas de investigación muy focalizadas en temáticas en las que dichos factores se encuentran presentes.

Una vez realizada una primera aproximación a los factores recogidos en el conjunto de referencias, es posible profundizar en los resultados obtenidos en la fase I, evaluando el grado en el que los diferentes factores contribuyen a cada uno de los enfoques con que se evalúa la figura del peatón. Así pues, el Gráfico 3 muestra cómo para el enfoque peatón-transporte, los factores más destacados son la "distancia total de la ruta" (79\%) y los "usos del suelo" (50\%), mientras que el resto de factores presentes en dichos artículos se mantiene en niveles inferiores al $20 \%$. Por el contrario, el enfoque peatón-entorno posee un mayor aporte de factores, con una presencia más homogénea, en la que destacan algunos como "usos del suelo", "iluminación", "tipología de acera", "volumen de tráfico", "arbolado", "mantenimiento de la acera", etcétera.

Por su parte, el enfoque mixto presenta un esquema intermedio entre los dos anteriores. Así pues, presenta el factor "usos del suelo" como el más relevante, estando presente prácticamente en el $90 \%$ de las referencias de este grupo. Además, da cuenta de otros factores, pero con menor relevancia, como, por ejemplo, el "arbolado" (47\%), el "diseńo de intersecciones" (47\%) o la "distancia total de la ruta" (37\%).

Una vez establecida la frecuencia de aparición de los diferentes factores en cada uno de los tres enfoques de evaluación de la figura del peatón, llama la atención cómo a priori podría parecer extraño que, en el enfoque peatón-transporte, el factor "usos del suelo" tenga menor presencia en las referencias que en los otros dos enfoques. Esta situación tiene lugar por la consideración de algunas referencias con enfoque peatón-transporte que focalizan su análisis en la distancia hacia un uso determinado, pero sin considerar los usos del suelo como determinantes en el análisis de la accesibilidad peatonal. Dichas referencias no consideran los usos del suelo como un factor en sí mismo. Por otra parte, hay que tener en cuenta la forma en que dicho factor es analizado en los distintos enfoques, ya que mientras en el enfoque peatóntransporte se analiza mayoritariamente de manera cuantitativa, en el enfoque peatónentorno y en el enfoque mixto, los usos del suelo son analizados mayoritariamente desde un punto de vista cualitativo.

Finalmente, esta distribución de factores entre los diferentes enfoques, así como su frecuencia de aparición en el conjunto de referencias, da una idea de cómo el 
enfoque mixto se sitúa como aquel que pretende responder a la necesidad de accesibilidad peatonal a diferentes usos del suelo, enriqueciendo el análisis respecto del enfoque más tradicional de accesibilidad peatonal. No obstante, este enfoque mixto supone también un ahorro en la consideración de factores respecto al enfoque peatónentorno, lo que lo convierte en una aproximación sintética y aglutinadora de mayor operatividad para la planificación de la movilidad peatonal en entornos urbanos.

\section{GRÁfICO 3 Representatividad de los factores según enfoque (\% de aparición del factor respecto del total de referencias del enfoque)}

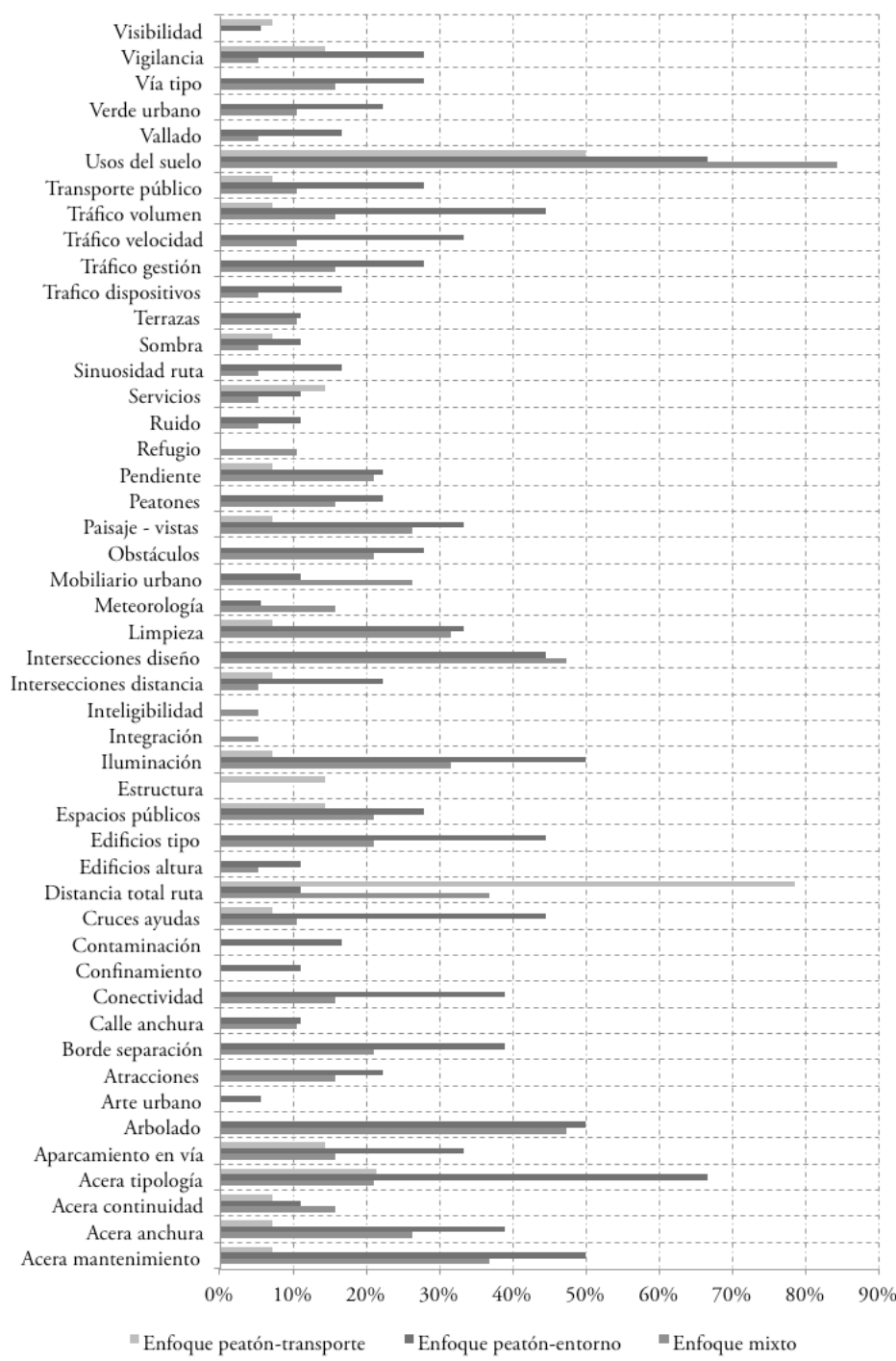


Analizados los enfoques de las publicaciones revisadas, así como los principales factores dentro de cada uno de esos enfoques, resulta interesante diferenciar dichos factores en función de la dimensión principal a la que pertenecen, ya sea esta morfológica, funcional o ambiental (Cuadro 3). Profundizando en los factores que forman parte de la dimensión morfológica de la calle, se constata que contribuyen en mayor o menor medida a sus aspectos físicos o infraestructurales, mientras que los factores de dimensión funcional son aquellos que le dan vitalidad a ese espacio en función de su densidad y diversidad. Finalmente, la dimensión ambiental recoge los factores que contribuyen a generar percepciones positivas en los peatones o satisfacer pequeñas necesidades durante el recorrido y, en definitiva, a crear espacios agradables para ellos.

En esta línea, y analizando de manera conjunta la frecuencia de los factores por enfoques y las dimensiones de dichos factores, se observa cómo el factor más determinante del enfoque peatón-transporte -distancia total de la ruta- tiene una dimensión morfológica. Por su parte, en el enfoque peatón-entorno, al recoger un gran número de factores, están presentes las tres dimensiones, aunque destacan, por la frecuencia de los factores, la dimensión funcional y la morfológica. Finalmente, en el enfoque mixto destaca la dimensión funcional como la más relevante, según el factor más representativo de dicho enfoque.

CUADro 3 Enfoque y dimensión de los factores recogidos en las referencias

\begin{tabular}{|c|c|c|c|c|c|c|c|}
\hline \multirow{2}{*}{ FACTOR } & \multicolumn{3}{|c|}{ DIMENSIÓN } & \multirow{2}{*}{ FACTOR } & \multicolumn{3}{|c|}{ DIMENSIÓN } \\
\hline & $\begin{array}{c}\text { MORFO- } \\
\text { LÓGICA }\end{array}$ & $\begin{array}{c}\text { FUNCIO- } \\
\text { NAL }\end{array}$ & $\begin{array}{c}\text { AMBIEN- } \\
\text { TAL }\end{array}$ & & $\begin{array}{l}\text { MORFO- } \\
\text { LÓGICA }\end{array}$ & $\begin{array}{c}\text { FUNCIO- } \\
\text { NAL }\end{array}$ & $\begin{array}{l}\text { AMBIEN- } \\
\text { TAL }\end{array}$ \\
\hline Acera mantenimiento & $\bullet$ & & & Meteorología & & & • \\
\hline Acera anchura & $\bullet$ & & & Mobiliario urbano & & & $\bullet$ \\
\hline Acera continuidad & $\bullet$ & & & Obstáculos & & & • \\
\hline Acera tipología & • & & & Paisaje & & & • \\
\hline Aparcamientos en vía & & $\bullet$ & & Peatones & & $\bullet$ & \\
\hline Arbolado & & & - & Pendiente & $\bullet$ & & \\
\hline Arte urbano & & & - & Refugio & & & - \\
\hline Atracciones & & - & & Ruido & & & • \\
\hline Borde separación & $\bullet$ & & & Señalización & & $\bullet$ & \\
\hline Calle anchura & $\bullet$ & & & Servicios & & • & \\
\hline Conectividad & $\bullet$ & & & Sinuosidad ruta & $\bullet$ & & \\
\hline Confinamiento & & & - & Sombras & & & $\bullet$ \\
\hline Contaminación & & & $\bullet$ & Terrazas & & $\bullet$ & \\
\hline Cruces ayudas & & & - & Tipo de calle & & $\bullet$ & \\
\hline Distancia total ruta & $\bullet$ & & & Tráfico dispositivo & & • & \\
\hline
\end{tabular}

(continúa) 
(continuación)

\begin{tabular}{|c|c|c|c|c|c|c|c|}
\hline \multirow[b]{2}{*}{ FACTOR } & \multicolumn{3}{|c|}{ DIMENSIÓN } & \multirow[b]{2}{*}{ FACTOR } & \multicolumn{3}{|c|}{ DIMENSIÓN } \\
\hline & $\begin{array}{c}\text { MORFO- } \\
\text { LÓGICA }\end{array}$ & $\begin{array}{c}\text { FUNCIO- } \\
\text { NAL }\end{array}$ & $\begin{array}{c}\text { AMBIEN- } \\
\text { TAL }\end{array}$ & & $\begin{array}{l}\text { MORFO- } \\
\text { LÓGICA }\end{array}$ & $\begin{array}{c}\text { FUNCIO- } \\
\text { NAL }\end{array}$ & $\begin{array}{c}\text { AMBIEN- } \\
\text { TAL }\end{array}$ \\
\hline Edificios-alturas & & $\bullet$ & & Tráfico-gestión & & $\bullet$ & \\
\hline Edificios-tipos & & • & & Tráfico-velocidad & & - & \\
\hline Espacio público & & $\bullet$ & & Tráfico-volumen & & $\bullet$ & \\
\hline Estructura & $\bullet$ & & & Transporte público & & $\bullet$ & \\
\hline Iluminación & & & $\bullet$ & Usos del suelo & & - & \\
\hline Integración & $\bullet$ & & & Vallado & & & - \\
\hline Inteligibilidad & $\bullet$ & & & Verde urbano & & & - \\
\hline Intersecciones distancia & $\bullet$ & & & Vía tipo & & $\bullet$ & \\
\hline Intersecciones diseño & $\bullet$ & & & Vigilancia & & & - \\
\hline $\begin{array}{l}\text { Limpieza-manteni- } \\
\text { miento }\end{array}$ & & & • & Visibilidad & $\bullet$ & & \\
\hline
\end{tabular}

FUENTE ELABORACIÓN PROPIA

En este contexto, se puede apreciar de manera temporal cómo los diferentes factores han contribuido a las tres dimensiones (Gráfico 4). Así pues, en el primer periodo tiene mayor relevancia la dimensión morfológica y ambiental. No obstante, esta relevancia decae en el siguiente periodo para ambas dimensiones, hecho que contrasta con lo que ocurre con la dimensión funcional, que tiene un fuerte incremento. Estas fluctuaciones en la distribución de factores en las diferentes dimensiones se estabilizan levemente en los siguientes periodos, aunque con una caída leve de la dimensión ambiental respecto a las otras dos dimensiones.

\section{GRÁFICO 4| Distribución temporal del número de factores general y por dimensiones}

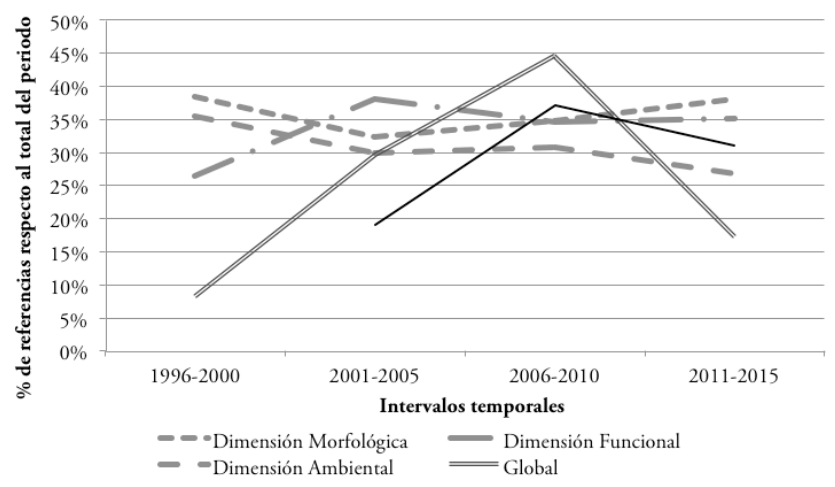

FUENTE ELABORACIÓN PROPIA

Si bien esta evolución temporal de las dimensiones a lo largo del periodo abarcado resulta de interés para conocer la importancia que se les ha otorgado en las investigaciones a lo largo del tiempo, también es interesante comprender el contexto global 
en el análisis de los factores. En este sentido, se puede apreciar cómo hasta el periodo 2006-2010 se da un fuerte crecimiento en los factores considerados en relación con la movilidad peatonal; sin embargo -y teniendo en cuenta que el último periodo de tiempo considerado todavía no ha concluido-, parece vislumbrarse un cambio de tendencia, en el que hay un decrecimiento en el número de factores considerados.

Por otra parte, y desde un punto de vista general, considerando la frecuencia de los factores según los distintos enfoques, así como la dimensión a la que contribuyen de manera más predominante, en el enfoque peatón-transporte la dimensión predominante es la morfológica, seguida de la funcional y, en último lugar, de la dimensión ambiental (Gráfico 5). En el caso del enfoque peatón-entorno y en el enfoque mixto la distribución de las dimensiones varía, siendo más predominante la dimensión funcional, seguida de las dimensiones morfológica y ambiental.

\section{GRÁfICO 5 Distribución de las dimensiones según frecuencia de los factores en los distintos enfoques}

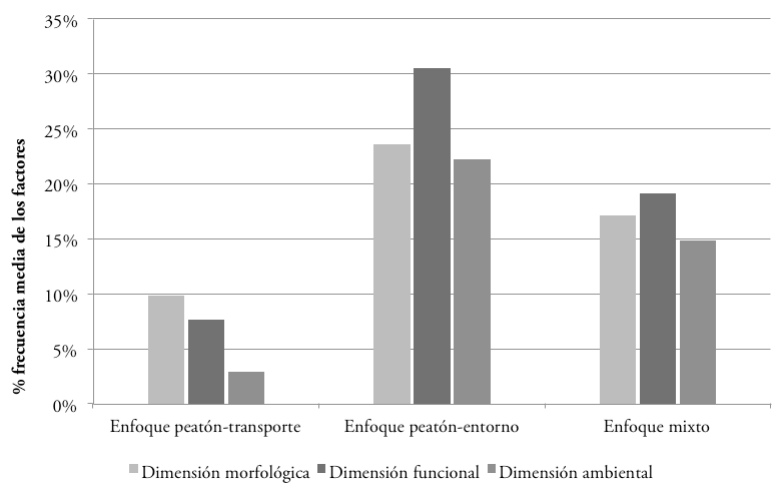

FUENTE ELABORACIÓN PROPIA

Finalmente, esta evaluación de los principales factores en cada uno de los enfoques y de las dimensiones dominantes que ha considerado esta revisión, según la frecuencia con las que están presentes en el conjunto de referencias, debe complementarse con un análisis de la valoración que posee en sí mismo el factor en cuestión respecto a los demás, de forma que esta valoración recoja la opinión o visión de los propios peatones.

Fase III: Valoración de los factores urbanos en relación con la movilidad peatonal A objeto de evaluar la calidad peatonal en un ámbito geográfico específico, es preciso no solo diseccionar la(s) relación(es) que se establece(n) entre los peatones y el entorno urbano por el que transitan, sino conocer también qué factores son los que propician o no la movilidad peatonal. Con esta finalidad se analizan los artículos que incluyen una valoración de los factores de diseño urbano por medio de encuestas a la población. En esta línea, los artículos seleccionados para realizar la comparativa de los factores considerados y su valoración por parte de la población como predominantes y, por ende, condicionantes, son seis (Cuadro 4), en los que se puede constatar diferentes enfoques y perspectivas. Por una parte, en lo que respecta 
al enfoque temático, existen a grandes rasgos dos enfoques: la perspectiva del diseño urbano, y otra más concerniente a la salud de la población. Por otra parte, existen diferencias claras en los sujetos que valoran los factores recogidos en las encuestas propuestas por cada uno de los autores. Así, dos de las seis referencias evaluadas establecen valoración de factores urbanos mediante un panel de expertos, mientras que en las cuatro referencias restantes, la valoración se establece con base en las respuestas de la población residente. En este último caso es posible distinguir a su vez pequeños matices que deben ser tenidos en cuenta, ya que de las cuatro referencias basadas en cuestionarios a población, Borst et al. (2009) se centran en la población de mayor edad y Weinstein et al. (2008) inciden en la población residente que es usuaria del transporte público.

CUADRO 4 Referencias seleccionadas para la valoración de factores

\begin{tabular}{|c|c|c|c|}
\hline REFERENCIA & $\begin{array}{l}\text { TEMÁTICA } \\
\text { REVISTA }\end{array}$ & COLECTIVO ENCUESTADO & ZONA DE ESTUDIO \\
\hline Adkins et al. (2012) & Diseño urbano & Población residente general & Portland (Oregón) \\
\hline Borst et al. (2009) & Psicología & Población residente anciana & $\begin{array}{l}\text { Schiedan, Holanda Meridional } \\
\text { (Holanda) }\end{array}$ \\
\hline $\begin{array}{l}\text { Ewing \& Handy } \\
\text { (2009) }\end{array}$ & Diseño urbano & Panel de expertos & $\begin{array}{l}\text { Multitud de ciudades (Estados } \\
\text { Unidos) }\end{array}$ \\
\hline Leslie et al. (2005) & Salud & Población residente general & $\begin{array}{l}\text { Adelaida, Australia Meridional } \\
\text { (Australia) }\end{array}$ \\
\hline Pikora et al. (2003) & $\begin{array}{l}\text { Ciencias sociales } \\
\text { y Medicina }\end{array}$ & Panel de expertos & ( \\
\hline $\begin{array}{l}\text { Weinstein et al. } \\
(2008)\end{array}$ & Diseño urbano & $\begin{array}{l}\text { Población usuaria transporte } \\
\text { público }\end{array}$ & $\begin{array}{l}\text { California y Oregón (Estados } \\
\text { Unidos) }\end{array}$ \\
\hline
\end{tabular}

FUENTE ELABORACIÓN PROPIA

Entrando en detalle en la valoración de factores recogidos en este subconjunto de referencias bibliográficas seleccionadas, el número de factores evaluados se ha reducido levemente desde los 48 factores de la fase II a 41 factores (Gráfico 6). De este conjunto de factores valorados, se puede apreciar cómo el factor "usos y negocios" ocupa un papel destacado de acuerdo con la frecuencia de aparición $(\mathrm{n}=6)$ respecto al total de referencias. A este factor le suceden otros cuya presencia en el conjunto de referencias es elevado ( $n=5)$, tal y como sucede con "acera-acondicionamiento", "arbolado", "edificios-tipos", la presencia de "espacios públicos" y el factor "paisaje", entendido este como paisaje natural y como paisaje urbano.

No obstante, la frecuencia de aparición de los factores en el conjunto de referencias no debe confundirse con su peso dentro del espectro de factores que pueden influir en la movilidad peatonal. Es por ello que, además de su frecuencia, es necesario caracterizar su intensidad a través de los valores obtenidos de las diferentes encuestas, ya sean estas a población o a expertos. En este contexto, y dado que cada uno de los autores ha realizado valoraciones distintas, es fundamental homogeneizar los factores y sus valores para que puedan ser comparados. Este proceso de homogeneización contiene ciertas licencias a la hora de agrupar factores y sus valoraciones, por lo que los resultados deben ser entendidos como relativos y establecidos desde un enfoque exploratorio. 


\section{GRÁFICO 6 | Valoración media de los factores en la movilidad peatonal}

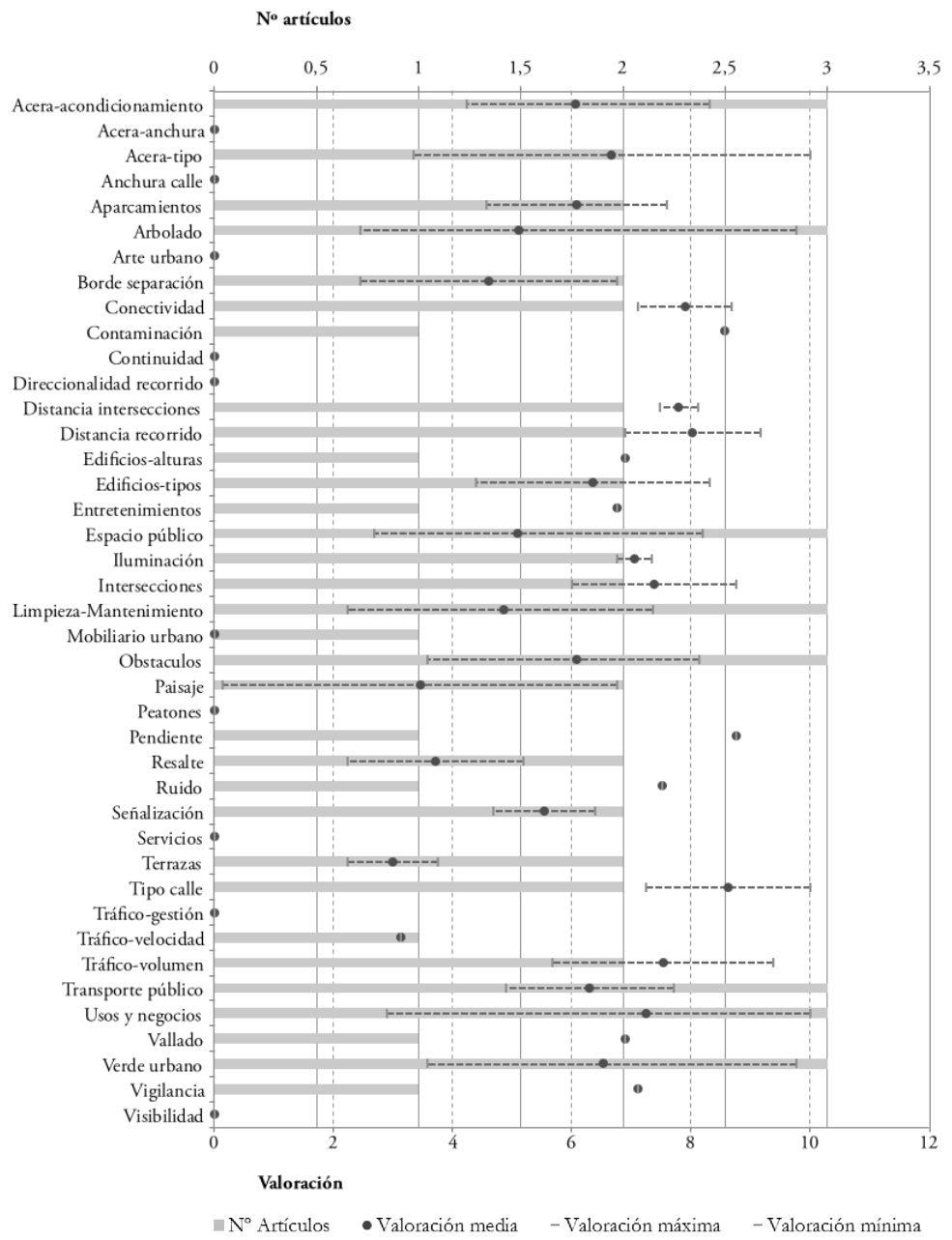

FUENTE ELABORACiÓN PROPIA A PARTIR DE ADKINS ET AL. (2OI2); BORST ET AL. (2009); EWING \& HANDY (2009); LESLIE ET AL. (2005); PIKORA ET AL. (2003); WEINSTEIN ET AL. (2008)

En el marco señalado, la valoración de los factores se ha homogeneizado en una escala de 0 a 10 , siendo el 0 el valor de menor influencia o peso dentro de los factores analizados en la movilidad peatonal y 10 el máximo factor de influencia o peso en dicha movilidad. Así pues, se puede apreciar en el Gráfico 6 los aspectos descritos anteriormente: en el eje superior y con barras grises estaría identificada la frecuencia de aparición de los factores en las referencias, y en el eje inferior y con puntos, la valoración media obtenida tras la homogeneización, así como los valores máximos y mínimos registrados. Ambos aspectos se exponen de manera conjunta, ya que integrando frecuencia e intensidad es posible tener una idea más ajustada de la valoración que se lleva a cabo para cada uno de los factores. 
Una vez puntualizados los aspectos anteriores, el análisis de la valoración de los factores muestra una valoración alta de factores como la "distancia recorrido" $(8,68)$, "vigilancia" $(8,56)$, "conectividad" $(7,89)$, "tipo de calle" $(7,57)$ o "intersecciones" $(7,37)$. De este grupo de factores con alta valoración media, es necesario atender en primer lugar a su frecuencia de aparición respecto al total de referencias analizadas en esta fase. De esta forma, se aprecia cómo la "distancia de recorrido" está presente en la mitad de las referencias, con una desviación típica de 1,32 puntos. Por su parte, los factores "intersecciones" y "tipo de calle" poseen frecuencias iguales al anterior factor descrito; sin embargo, sus desviaciones típicas varían del 1,12 al 1,86, respectivamente, lo que indicaría un menor consenso en las respuestas obtenidas. Los factores "conectividad" y "vigilancia" ostentan, pese a su elevado valor medio, una frecuencia de aparición menor, aunque con desviaciones típicas de 0,78 y 1,45, respectivamente.

En la franja de valoración media se sitúan factores como "tráfico-gestión” (6,89), "iluminación” (6,81), “acera-tipo" (6,63), “tráfico-volumen” (6,42), “distancia intersecciones" $(6,37)$ u "obstáculos" $(6,07)$. De este conjunto de factores cabe hacer especial mención del factor "iluminación”, considerando su frecuencia respecto a la segunda selección de referencias: se encuentra en cuatro de las seis referencias evaluadas, aunque no existe un elevado consenso en su valoración, ya que muestra una desviación típica de 2,17. Esta situación de elevada frecuencia, y una desviación típica mayor a la del grupo de factores anteriores, es la tónica general para este grupo de factores, a excepción del factor "gestión de tráfico", cuya frecuencia y desviación típica se ven reducidas a 2 y 0,18 , respectivamente.

\section{Conclusiones}

El presente artículo pone de relieve la complejidad y diversidad de funciones del peatón en el medio urbano, lo que genera la necesidad de conocer en profundidad las características del entorno construido que más influyen en el peatón a la hora de desplazarse o acceder a distintas centralidades urbanas. De ahí que los resultados que arroja la metodología de revisión contribuyan a caracterizar estos ambientes o entornos peatonales para conocer mejor sus cualidades y condicionantes a la hora de favorecer la accesibilidad al espacio público a escala de planificación y de diseño urbano.

La riqueza de factores identificados mediante la exploración bibliográfica es ya una revelación de la necesidad de revisar tanto los enfoques como las dimensiones a la hora de analizar, proyectar y evaluar la accesibilidad peatonal, como objetivo de integración y calidad urbana basado en la interdependencia y jerarquía de factores. En este sentido, la primera fase de la metodología evidencia cómo el análisis del peatón ha evolucionado a lo largo de las dos últimas décadas desde enfoques más específicos y deterministas, hacia un enfoque más holístico o mixto. Esta evolución pone de manifiesto la necesidad de considerar enfoques y modelos integrados que aporten una nueva visión de conjunto ante la complejidad propia de la figura del peatón. Solo así será posible desarrollar posteriormente herramientas útiles en los procesos de planificación y gestión de la movilidad peatonal, máxime si esta quiere ser entendida como una movilidad importante en el intercambio modal, por ejemplo, mediante la combinación de factores favorables a la proximidad urbana. 
Además, el referido enfoque mixto para el análisis de la movilidad y accesibilidad peatonal en entornos urbanos debe, en última instancia, crear para el peatón un fuerte "sentido de lugar", considerando y equilibrando los factores que intervienen dentro de las dimensiones morfológica, funcional y ambiental. No obstante, tal como se ha puesto de manifiesto en la tendencia de los factores en el marco temporal analizado, el número de factores por considerar debe ser -en la medida de lo posible- reducido; deben aportar riqueza a los análisis y ser, al mismo tiempo, fácilmente manejables por parte del conjunto de actores concernidos, sin necesidad de elevados conocimientos al respecto. Por consiguiente, la riqueza de factores no significa una multiplicación innecesaria en el momento de su identificación relevante, sino especificidad en su manejo según el enfoque idóneo y/o dominante con el cual favorecer la accesibilidad peatonal en un entorno determinado.

En este contexto, los factores que han revelado una mayor representatividad dentro del conjunto considerado para la movilidad y accesibilidad peatonal han sido la "distancia hasta destino", los "usos del suelo", el "tipo de acera" o el "arbolado". Por tanto, a la hora de analizar entornos urbanos en los que mejorar la calidad de la movilidad y accesibilidad peatonal, estos factores deben estar muy presentes. Lógicamente, la tendencia observada es el resultado de una revisión no exhaustiva y, por tanto, es limitado el número considerado de referencias según el proceso metodológico expuesto. Puede, entonces, ser sin duda enriquecedora y esclarecedora una revisión más extensa, incluyendo otras revistas y bases de datos para ratificar la representatividad de estos factores.

En relación con lo anterior, es necesario enfatizar que, tal y como se plantea en la discusión sobre la valoración de los factores urbanos en relación con la movilidad peatonal, la frecuencia de aparición de los factores en el conjunto de referencias no debe confundirse con su peso dentro del espectro de factores que pueden influir en la movilidad peatonal, ya que no dejan de ser valores relativos desde un enfoque exploratorio. En consecuencia, además de considerar aquellos factores que frecuentemente son utilizados en las diferentes investigaciones sobre la movilidad o la accesibilidad peatonal, es necesario conocer de qué manera dichos factores condicionan más a los peatones a la hora de desplazarse a pie. Con esta finalidad es posible jerarquizar los diferentes factores urbanos según la importancia que les otorga la población o un panel de expertos a través de encuestas. Como se ha puesto de manifiesto en la fase III del presente artículo, la valoración de los distintos factores por parte de la población pone de manifiesto la importancia de considerar la escala local cuando se analiza la figura del peatón, ya que la valoración de los factores no es extrapolable. Este hecho evidencia la relación directa que mantiene el peatón con el entorno, donde la climatología, la cultura, etcétera, desempeñan un importante papel.

Tras esta aproximación o revisión global, aunque también pormenorizada y representativa de los enfoques y factores relacionados con los desplazamientos peatonales, se abren líneas futuras de investigación. Ellas se basan, por un lado, en un fortalecimiento de la fundamentación metodológica mediante la extensión de las referencias consideradas; y, por otro lado, en una evaluación de la percepción que poseen los peatones respecto de los distintos factores en un contexto específico, en consonancia con la relevancia identificada de enfoques, dimensiones y factores. 


\section{Agradecimientos}

Este trabajo ha sido elaborado con la financiación de la Junta de Andalucía y el Fondo Europeo de Desarrollo Regional (FEDER), en el marco del Proyecto de Excelencia P09-RNM-5394: "Guía Metodológica para la Integración Metropolitana Sostenible de los Sistemas de Metro Ligero (INTEGRA-ME)”.

\section{Referencias bibliográficas}

Adkins, A., Dill, J., Luhr, G. \& Neal, M. (2012). Unpacking walkability: Testing the influence of urban design features on perceptions of walking environment attractiveness. Journal of Urban Design, 17(4), 499-510. doi: 10.1080/13574809.2012.706365

Alfonzo, M., Boarnet, M. G., Day, K., McMillan, T. \& Anderson, C. L. (2008). The relationship of neighbourhood built environment features and adult parents' walking. Journal of Urban Design, 13(1), 29-51.

Alfonzo, M. A. (2005). To walk or not to walk? The hierarchy of walking needs. Environment and Behavior, 37(6), 808-836. doi: 10.1177/0013916504274016

Apparicio, P. \& Seguin, A.-M. (2006). Measuring the accessibility of services and facilities for residents of public housing in Montreal. Urban Studies, 43(1), 187-211. doi: 10.1080/00420980500409334

Bentley, R., Jolley, D. \& Kavanagh, A. M. (2010). Local environments as determinants of walking in Melbourne, Australia. Social Science \& Medicine, 70(11), 1806-1815. http://dx.doi.org/10.1016/j.socscimed.2010.01.041

Boarnet, M. G., Joh, K., Siembab, W., Fulton, W. \& Mai Thi Nguyen. (2011). Retrofitting the suburbs to increase walking: Evidence from a land-use-travel study. Urban Studies, 48(1), 129-159. doi: 10.1177/0042098010364859

Borst, H. C., de Vries, S. I., Graham, J. M. A., van Dongen, J. E. F., Bakker, I. \& Miedema, H. M. E. (2009). Influence of environmental street characteristics on walking route choice of elderly people. Journal of Environmental Psychology, 29(4), 477-484.

Bricka, S., Sener, I., Dusza, C., Wood, N. \& Hudson, J. (2012). Factors influencing walking in small urban region. Transportation Research Record: Journal of the Transportation Research Board, 2307(-1), 52-59. doi: 10.3141/2307-06

Cao, X., Handy, S. \& Mokhtarian, P. (2006). The influences of the built environment and residential self-selection on pedestrian Behavior: Evidence from Austin, TX. Transportation, 33(1), 1-20. doi: 10.1007/s11116-005-7027-2

Cervero, R. \& Kockelman, K. (1997). Travel demand and the 3Ds: Density, diversity, and design. Transportation Research Part D: Transport and Environment, 2(3), 199-219. http://dx.doi.org/10.1016/S1361-9209(97)00009-6

Clifton, K. J., Livi Smith, A. D. \& Rodriguez, D. (2007). The development and testing of an audit for the pedestrian environment. Landscape and Urban Planning, 80(1-2), 95110. http://dx.doi.org/10.1016/j.landurbplan.2006.06.008

Correa-Díaz, G. (2010). Transporte y ciudad. EURE, 36(107), 133-137. http://dx.doi. org/10.4067/S0250-71612010000100008 
Dávila, J. D. (2012). Nuevos transportes y movilidad urbana. Bitácora urbano territorial, 21(2), 57-60.

Dixon, L. (1996). Bicycle and pedestrian level-of-service performance measures and standards for congestion management systems. Transportation Research Record: Journal of the Transportation Research Board, 1538(-1), 1-9. http://dx.doi.org/10.3141/1538-01

Ewing, R. \& Handy, S. (2009). Measuring the unmeasurable: urban design qualities related to walkability. Journal of Urban Design, 14(1), 65 - 84. doi: 10.1080/13574800802451155

Foltête, J.-C. \& Piombini, A. (2007). Urban layout, landscape features and pedestrian usage. Landscape and Urban Planning, 81(3), 225-234. http://dx.doi.org/10.1016/j. landurbplan.2006.12.001

Foster, S., Giles-Corti, B. \& Knuiman, M. (2011). Creating safe walkable streetscapes: Does house design and upkeep discourage incivilities in suburban neighbourhoods? Journal of Environmental Psychology, 31(1), 79-88. http://dx.doi.org/10.1016/j. jenvp.2010.03.005

Gallimore, J. M., Brown, B. B. \& Werner, C. M. (2011). Walking routes to school in new urban and suburban neighborhoods: An environmental walkability analysis of blocks and routes. Journal of Environmental Psychology, 31(2), 184-191. doi: 10.1016/j. jenvp.2011.01.001

Gehl, J. (1971). Life between buildings: using public space: Copenhague: Danish Architectural Press.

Giles-Corti, B., Broomhall, M. H., Knuiman, M., Collins, C., Douglas, K., Ng, K., Lange, A., Donovan, R. J. (2005). Increasing walking: How important is distance to, attractiveness, and size of public open space? American Journal of Preventive Medicine, 28(2, Supplement 2), 169-176. doi: 10.1016/j.amepre.2004.10.018

Giles-Corti, B. \& Donovan, R. J. (2002). Socioeconomic Status Differences in Recreational Physical Activity Levels and Real and Perceived Access to a Supportive Physical Environment. Preventive Medicine, 35(6), 601-611. http://dx.doi.org/10.1006/ pmed.2002.1115

Guo, Z. (2009). Does the pedestrian environment affect the utility of walking? A case of path choice in downtown Boston. Transportation Research Part D: Transport and Environment, 14(5), 343-352. http://dx.doi.org/10.1016/j.trd.2009.03.007

Hernández, D. (2012). Activos y estructuras de oportunidades de movilidad. Una propuesta analítica para el estudio de la accesibilidad por transporte público, el bienestar y la equidad. EURE, 38(115), 117-135. http://dx.doi.org/10.4067/S025071612012000300006

Iacono, M., Krizek, K. J. \& El-Geneidy, A. (2010). Measuring non-motorized accessibility: issues, alternatives, and execution. Journal of Transport Geography, 18(1), 133-140. http://dx.doi.org/10.1016/j.jtrangeo.2009.02.002

Jacobs, A. B. (1993). Great Streets. Cambridge, ma: The mIt Press.

Jiang, Y., Christopher Zegras, P. \& Mehndiratta, S. (2012). Walk the line: station context, corridor type and bus rapid transit walk access in Jinan, China. Journal of Transport Geography, 20(1), 1-14. http://dx.doi.org/10.1016/j.jtrangeo.2011.09.007

Kelly, C. E., Tight, M. R., Hodgson, F. C. \& Page, M. W. (2011). A comparison of three methods for assessing the walkability of the pedestrian environment. Journal of Transport Geography, 19(6), 1500-1508. http://dx.doi.org/10.1016/j.jtrangeo.2010.08.001 
Koh, P. P. \& Wong, Y. D. (2013). Comparing pedestrians' needs and behaviours in different land use environments. Journal of Transport Geography, 26(0), 43-50. http://dx.doi. org/10.1016/j.jtrangeo.2012.08.012

Landis, B., Vattikuti, V., Ottenberg, R., McLeod, D. \& Guttenplan, M. (2001). Modeling the roadside walking environment: Pedestrian level of service. Transportation Research Record: Journal of the Transportation Research Board, 1773(-1), 82-88. doi: $10.3141 / 1773-10$

Learnihan, V., Van Niel, K. P., Giles-Corti, B. \& Knuiman, M. (2011). Effect of scale on the links between walking and urban design. Geographical Research, 49(2), 183-191. doi: 10.1111/j.1745-5871.2011.00689.x

Lee, C. \& Moudon, A. V. (2006). The 3Ds+R: Quantifying land use and urban form correlates of walking. Transportation Research Part D: Transport and Environment, 11(3), 204215. doi: 10.1016/j.trd.2006.02.003

Lee, R. E., Mama, S. K., Medina, A. V., Ho, A. \& Adamus, H. J. (2012). Neighborhood factors influence physical activity among African American and Hispanic or Latina women. Health \& Place, 18(1), 63-70. doi: 10.1016/j.healthplace.2011.08.013

Lee, S., Son, H. \& Joo, Y. (2012). A new approach for the evaluation of the walking environment. International Journal of Sustainable Transportation, 7(3), 238-260. doi: 10.1080/15568318.2013.710146

Leslie, E., Saelens, B., Frank, L., Owen, N., Bauman, A., Coffee, N. \& Hugo, G. (2005). Residents' perceptions of walkability attributes in objectively different neighbourhoods: a pilot study. Health \& Place, 11(3), 227-236. http://dx.doi.org/10.1016/j. healthplace.2004.05.005

Litman, T. (2003). Economic value of walkability. Transportation Research Record: Journal of the Transportation Research Board, 1828(1), 3-11. En http://atfiles.org/files/pdf/ walkability.pdf

Lotfi, S. \& Koohsari, M. J. (2009). Measuring objective accessibility to neighborhood facilities in the city (A case study: Zone 6 in Tehran, Iran). Cities, 26(3), 133-140. http:// dx.doi.org/10.1016/j.cities.2009.02.006

Loutzenheiser, D. (1997). Pedestrian access to transit: model of walk trips and their design and urban form determinants around Bay area rapid transit stations. Transportation Research Record: Journal of the Transportation Research Board, 1604(-1), 40-49. http:// dx.doi.org/10.3141/1604-06

Lynch, K. (1960/1981). La imagen de la ciudad (E. L. Revol, Trad.). Barcelona: Gustavo Gili.

Manaugh, K. \& El-Geneidy, A. (2011). Validating walkability indices: How do different households respond to the walkability of their neighborhood? Transportation Research Part D: Transport and Environment, 16(4), 309-315. http://dx.doi.org/10.1016/j. $\operatorname{trd} .2011 .01 .009$

McCormack, G. R., Cerin, E., Leslie, E., Du Toit, L. \& Owen, N. (2008). Objective versus perceived walking distances to destinations - Correspondence and predictive validity. Environment and Behavior, 40(3), 401-425. doi: 10.1177/0013916507300560

Millington, C., Ward Thompson, C., Rowe, D., Aspinall, P., Fitzsimons, C., Nelson, N. \& Mutrie, N. (2009). Development of the Scottish Walkability Assessment Tool (swat). Health \& Place, 15(2), 474-481. http://dx.doi.org/10.1016/j.healthplace.2008.09.007 
Moniruzzaman, M. \& Paez, A. (2012). A model-based approach to select case sites for walkability audits. Health \& Place, 18(6), 1323-1334. doi: 10.1016/j.healthplace.2012.09.013

Montgomery, J. (1998). Making a city: Urbanity, vitality and urban design. Journal of Urban Design, 3(1), 93-116. doi: 10.1080/13574809808724418

Neutens, T., Schwanen, T., Witlox, F. \& De Maeyer, P. (2010). Equity of urban service delivery: a comparison of different accessibility measures. Environment and Planning A, 42(7), 1613-1635. http://dx.doi.org/10.1068/a4230

O’Sullivan, S. \& Morrall, J. (1996). Walking distances to and from light-rail transit stations. Transportation Research Record: Journal of the Transportation Research Board, 1538(-1), 19-26. doi: 10.3141/1538-03

Olszewski, P. \& Wibowo, S. (2005). Using equivalent walking distance to assess pedestrian accessibility to transit stations in Singapore. Transportation Research Record: Journal of the Transportation Research Board, 1927(-1), 38-45. doi: 10.3141/1927-05

Owen, N., Humpel, N., Leslie, E., Bauman, A. \& Sallis, J. F. (2004). Understanding environmental influences on walking: Review and research agenda. American Journal of Preventive Medicine, 27(1), 67-76. http://dx.doi.org/10.1016/j.amepre.2004.03.006

Parks, J. R. \& Schofer, J. L. (2006). Characterizing neighborhood pedestrian environments with secondary data. Transportation Research Part D: Transport and Environment, 11(4), 250-263. http://dx.doi.org/10.1016/j.trd.2006.04.003

Pasaogullari, N. \& Doratli, N. (2004). Measuring accessibility and utilization of public spaces in Famagusta. Cities, 21(3), 225-232. http://dx.doi.org/10.1016/j.cities.2004.03.003

Peters, P. (1981). La ciudad peatonal. 2a ed. Barcelona: Gustavo Gili.

Pikora, T., Giles-Corti, B., Bull, F., Jamrozik, K. \& Donovan, R. (2003). Developing a framework for assessment of the environmental determinants of walking and cycling. Social Science \& Medicine, 56(8), 1693-1703. http://dx.doi.org/10.1016/S02779536(02)00163-6

Pooley, C. G., Horton, D., Scheldeman, G., Tight, M., Jones, T., Chisholm, A., Harwattb, H., Jopson, A. (2011). Household decision-making for everyday travel: a case study of walking and cycling in Lancaster (UK). Journal of Transport Geography, 19(6), 16011607. http://dx.doi.org/10.1016/j.jtrangeo.2011.03.010

Rodríguez, D. A., Brisson, E. M. \& Estupinán, N. (2009). The relationship between segmentlevel built environment attributes and pedestrian activity around Bogota's BRT stations. Transportation Research Part D: Transport and Environment, 14(7), 470-478. doi: 10.1016/j.trd.2009.06.001

Shay, E., Rodriguez, D. A., Cho, G., Clifton, K. J. \& Evenson, K. R. (2009). Comparing objective measures of environmental supports for pedestrian travel in adults. International Journal of Health Geographics, 8. doi: 10.1186/1476-072x-8-62

Shriver, K. (1997). Influence of environmental design on pedestrian travel behavior in four Austin neighborhoods. Transportation Research Record: Journal of the Transportation Research Board, 1578(-1), 64-75. http://dx.doi.org/10.3141/1578-09

Talen, E. (2002). Pedestrian Access as a Measure of Urban Quality. Planning Practice \& Research, 17(3), 257-278. doi: 10.1080/026974502200005634

Talen, E. \& Anselin, L. (1998). Assessing spatial equity: an evaluation of measures of accessibility to public playgrounds. Environment and Planning A, 30(4), 595-613. doi: 10.1068/ a300595 
Townsend, C. \& Zacharias, J. (2010). Built environment and pedestrian behavior at rail rapid transit stations in Bangkok. Transportation, 37(2), 317-330. doi: 10.1007/s11116009-9226-8

Venturi, R., Brown, D. S. \& Izenour, S. (1977). Learning from Las Vegas: the forgotten symbolism of architectural form (Ed. revisada). Cambridge, MA: The MIT Press.

Weinstein, A., Schlossberg, M. \& Irvin, K. (2008). How Far, by Which Route and Why? A Spatial Analysis of Pedestrian Preference. Journal of Urban Design, 13(1), 81-98. doi: $10.1080 / 13574800701804074$

Werner, C. M., Brown, B. B. \& Gallimore, J. (2010). Light rail use is more likely on "walkable" blocks: Further support for using micro-level environmental audit measures. Journal of Environmental Psychology, 30(2), 206-214. http://dx.doi.org/10.1016/j. jenvp.2009.11.003

Wood, L., Frank, L. D. \& Giles-Corti, B. (2010). Sense of community and its relationship with walking and neighborhood design. Social Science \& Medicine, 70(9), 1381-1390. http://dx.doi.org/10.1016/j.socscimed.2010.01.021

World Health Organization (2011). Global strategy on diet, physical activity and health. Global Recommendations on Physical Activity for Health. En http://www.who.int/ dietphysicalactivity/en/

Zacharias, J. (2001). Pedestrian Behavior and Perception in Urban Walking Environments. Journal of Planning Literature, 16(1), 3-18. doi: 10.1177/08854120122093249

Zook, J. B., Lu, Y., Glanz, K. \& Zimring, C. (2012). Design and Pedestrianism in a Smart Growth Development. Environment and Behavior, 44(2), 216-234. doi: $10.1177 / 0013916511402060$ 
